\title{
Safety, Immunogenicity, and Transmissibility of Single-Dose Live Oral Cholera Vaccine Strain CVD 103-HgR in 24- to 59-Month-Old Indonesian Children
}

\author{
Cyrus H. Simanjuntak, Peter O'Hanley, \\ Narain H. Punjabi, Fernando Noriega, \\ Gary Pazzaglia, Patricia Dykstra, Bradford Kay, \\ Suharyono, Aswitha Budiarso, Atti R. Rifai, \\ Steven S. Wasserman, Genevieve Losonsky, \\ James Kaper, Stanley Cryz, and Myron M. Levine
}

\author{
National Institute of Health Research and Development, US Naval \\ Medical Research Unit No. 2, and Department of Pediatrics, University \\ of Indonesia, Jakarta, and Infectious Diseases Hospital, North Jakaria. \\ Indonesia; VA Hospital, Palo Alto, and Departments of Medicine and \\ Microbiology and Immunology. Stanford University School of Medicine. \\ Stanford. California; Center for Vaccine Development. University of \\ Maryland School of Medicine, Baltimore; and Swiss Serum and Vaccine \\ Institute, Berne, Switzerland
}

\begin{abstract}
Recombinant $\mathrm{A}^{-} \mathrm{B}^{+}$Vibrio cholerae $\mathrm{O} 1$ strain CVD $103-\mathrm{HgR}$ is a safe, highly immunogenic, single-dose live oral vaccine in adults in industrialized countries. Safety, excretion, immunogenicity, vaccine transmissibility, and environmental introduction of CVD $103-\mathrm{HgR}$ were investigated among 24- to 59-month-old children in Jakarta. In 81 households, 1 child was randomly allocated a single dose of vaccine $\left(5 \times 10^{9} \mathrm{cfu}\right)$ and another, placebo. Additionally, 139 unpaired children were randomly allocated vaccine or placebo. During 9 days of follow-up, diarrhea or vomiting did not occur more often among vaccinees than controls. Vaccine was minimally excreted and was isolated from no controls and from $1(0.6 \%)$ of 177 unvaccinated family contacts. A 4-fold or higher rise in serum vibriocidal antibody was observed in $75 \%$ of vaccinees (10-fold rise in geometric mean titer over baseline). Of 135 paired placebo recipients or household contacts, 5 had vibriocidal seroconversions. Moore swabs placed in sewers and latrines near 97 households failed to detect vaccine. These observations pave the way for a large-scale field trial of efficacy.
\end{abstract}

The return of cholera to South America in explosive epidemic form in 1991, after a century of absence [1], has focused attention on the lack of a satisfactory cholera vaccine. The venerable inactivated whole cell parenteral vaccine confers only limited $(\sim 50 \%)$ protection for a short time (generally 4-6 months) $[2,3]$. Consequently, public health authorities advise that the parenteral vaccine should play no role in the control of epidemic or endemic cholera $[4,5]$. In contrast, the World Health Organization has targeted the development of a new, improved cholera vaccine as a high priority $[6,7]$. To be a practical public health tool, a new

Received 25 March 1993; revised 7 July 1993.

Informed consent was obtained from parents or guardians and human experimentation guidelines of the US Department of Health and Human Services were followed. The protocol was reviewed by ethical committees at the University of Maryland School of Medicine, the National lnstitute of Health Research and Development, Jakarta (registered with the Office for the Protection of Research Risks, National Institutes of Health, Bethesda, MD), and the US Department of the Navy.

Financial support: US Agency for International Development; Consultative Group on Vaccine Development of the National Vaccine Program, United States; National Institute of Allergy and Infectious Diseases (contract AI-62528); US Naval Medical Research and Development Command (work unit no. 62787A.AN870.AN8).

Reprints or correspondence: Dr. M. M. Levine, Center for Vaccine Development, University of Maryland School of Medicine, 10 S. Pine St., Baltimore. MD 21201 .

The Journal of Infectious Diseases 1993;168:1169-76 (C) 1993 by The University of Chicago. All rights reserved 0022-1899/93/6805-0012\$01.00 cholera vaccine should be oral, single-dose, safe, and highly efficacious (even in young children) [8]. Moreover, ideally, protective efficacy should commence within a few days of administration and should persist long-term (at least 5 years).

Oral vaccines consisting of inactivated vibrios given with or without B subunit of cholera toxin conferred moderate (50\%-52\%) protection against cholera for 3 years in a field trial in Bangladesh $[9,10]$. While constituting an advance over the parenteral inactivated vaccine, the two oral inactivated vaccines tested in Bangladesh suffer from some notable drawbacks: Young children derived only short-term protection lasting < $<$ year [10]; protection in adults did not persist beyond 3 years; multiple (three, perhaps as few as two) spaced doses were necessary to confer protection $[9$, 10]; and protection was lower in persons of blood group $\mathrm{O}$ (an important host risk factor for cholera gravis) and against El Tor rather than classical biotype infection.

It is anticipated that genetically engineered strains of $\mathrm{Vib}$ rio cholerae $\mathrm{Ol}$ used as live oral vaccines may overcome the drawbacks of earlier generations of cholera vaccines and may, with a single dose, provide a high level of long-term protection among all age groups, without causing adverse reactions [8, 11-13]. However, the use of recombinant live vaccines raises certain other questions that must be answered, such as to what extent such strains are excreted, whether the vaccine strain is readily transmitted to contacts, and whether the vaccine persists in the environment. 
Strain CVD 103-HgR, presently the leading live oral cholera vaccine candidate, was derived from wild-type classical $V$. cholerae O1 569B (classic biotype, Inaba serotype) using recombinant DNA techniques [13-15]. Ninety-four percent of the gene encoding the A (ADP-ribosylating) subunit of cholera enterotoxin was deleted (leaving expression of the nontoxic $B$ subunit intact), and a gene encoding resistance to $\mathrm{Hg}^{++}$was inserted into the hlyA locus of the chromosome to provide a marker to differentiate CVD 103-HgR from wildtype vibrios [13-15]. The safety and immunogenicity of CVD $103-\mathrm{HgR}$ have been previously documented in randomized, placebo-controlled, double-blind clinical trials and open studies in adults in industrialized countries [14, 16-19] and adults and school-age children in developing countries [20-23]. Herein we report the first clinical studies of CVD $103-\mathrm{HgR}$ in preschool children in a less-developed country. In addition to safety and immunogenicity, excretion and transmissibility of the vaccine strain were investigated and bacteriologic studies were done to detect the vaccine strain in the environment around the dwellings of vaccinated subjects.

\section{Materials and Methods}

\section{Overview of the Studies}

El Tor cholera occurs throughout the year in Jakarta, with seasonal increases in June-August and in December and January [24, 25]. In July and August 1991, pairs of children 24-59 months of age within the same household were randomly allocated so that 1 child received, with buffer, a single dose of CVD $103-\mathrm{HgR}$ vaccine containing $5 \times 10^{9} \mathrm{cfu}$ while the other received placebo. A second round of vaccination was carried out in January and February 1992. Because few households with pairs of age-eligible children were available, for this round of vaccination the protocol was modified so that (except for a small number of household pairs) only I child 24-59 months old per household was entered into the study and was randomly allocated vaccine or placebo. Double-blind clinical surveillance detected adverse reactions, and a rise in serum vibriocidal antibody was the main outcome of immunogenicity. All participating children and many family members had multiple stool cultures to detect excretion of the vaccine strain. Blood specimens were obtained from most family members who gave stool cultures. In a proportion of households, environmental bacteriologic studies were carried out to detect the vaccine strain.

\section{Study Site}

The two studies were carried out in villages (kelurahans) in North Jakarta, located within the catchment area of the Infectious Diseases Hospital, North Jakarta.

\section{Subjects, Eligibility, Recruitment, and Allocation}

Study 1. With active participation of the kelurahan civic leaders, a census was undertaken to identify and describe the study to households with 2 children 24-59 months of age. Families who expressed an interest in participating were invited to a meeting where the study was again explained, questions were answered, and the vaccination schedule was given. Interested families who did not attend the meeting were sent an informational letter that included vaccination date and time and a consent form. For each household, one child was administered a coded preparation labeled $\mathrm{N}$ and the other $\mathrm{O}$ (one signified vaccine and the other placebo). The older child in each pair was inoculated first. The randomized pairs of coded preparations and lists denoting the preparation to be administered to each child were generated by the manufacturer. By maintaining bacteriologic and serologic surveillance of all subjects in this randomized, double-blind, placebo-controlled study, it was possible to detect evidence of transmission of the vaccine strain to placebo recipients.

Study 2. The experience in study $\mathrm{I}$ and subsequent censuses in other villages revealed that because of successful family planning programs, there were few households with pairs of children within the 24- to 59-month age group. Accordingly, the study design was modified to include households having 1 child 24-59 months old. Age-eligible children were recruited as in study 1. On the day of vaccination, after a physical examination and other measures to verify eligibility, age-eligible children from single-subject households were randomized to receive a number-coded preparation (about one-half of which corresponded to packets of vaccine and the remainder placebo). A separate set of packets was available for the few pairs that were recruited for the second study; allocation followed the procedure of the first study except that these packets had number codes.

Vaccination was carried out in the village office near a local health center. Children with chronic health problems, receiving antibiotic therapy, or with acute illness on the scheduled day of vaccination were excluded.

\section{Vaccines and Placebo}

Packets of vaccine contained $5 \times 10^{9}$ cfu of lyophilized, filtered CVD 103-HgR [22]. Placebo $\left(5 \times 10^{8}\right.$ inactivated Escherichia coli $\mathrm{K} 12$ organisms) was of the type extensively used in evaluation of inactivated and live oral cholera vaccines $[16,17$, 20-26]. Each packet of vaccine or placebo also contained 18.8 $\mathrm{mg}$ of aspartame to sweeten the vaccine cocktail and make it more palatable to children. The sachet of buffer that accompanied each packet of vaccine or placebo held $1.24 \mathrm{~g}$ of $\mathrm{NaHCO}_{3}$ and $0.88 \mathrm{~g}$ of ascorbic acid. The buffer powder was stirred into a cup containing $50 \mathrm{~mL}$ of nonchlorinated water, after which the vaccine (or placebo) packet was emptied into the buffer solution, stirred again, and immediately given to the child. Parents had been instructed that the children should refrain from eating or drinking for $\mathrm{l} h$ before and after vaccination. Children were considered vaccinated if they drank $\geqslant 50 \%$ of the suspension.

\section{Medical Supervision}

Physicians visited the children daily for 9 days in their households to elicit and document complaints, do physical examinations, and record axillary temperatures. The clinical follow-up, 
as well as the administration of vaccine (or placebo), was double-blind with neither the clinical staff, the children, nor their parents knowing the identity of the preparation given to any child. Diarrhea was defined as passage of at least three loose stools within $24 \mathrm{~h}$. Vomiting was defined as one or more episodes of emesis. An axillary temperature $\geqslant 37.5^{\circ} \mathrm{C}$ signified fever. Any child who passed three or more loose stools in a 24-h period was given oral rehydration with glucose and electrolytes.

\section{Bacteriology}

Clinical. Stool samples or rectal swabs were obtained daily from the children on the 5 days after vaccination. During study 1 , an attempt was also made to obtain stool or rectal swabs from family members in the households as a means of monitoring for transmission of the vaccine strain to contacts. Stool or swabs were transported to the laboratory in Cary-Blair medium and inoculated directly onto thiosulfate-citrate-bile salts-sucrose (TCBS) agar or were enriched for 5-6 h in alkaline peptone water before subculture onto TCBS agar [27]. Suspected colonies were confirmed as $V$. cholerae by biochemical tests and as $\mathrm{O}$ I serogroup by agglutination with specific antiserum. Serotyping was done by agglutination with specific Inaba and Ogawa antisera, and preliminary biotyping was based on resistance to polymyxin B (El Tor isolates are resistant, classical are not), hemagglutination of chicken erythrocytes (El Tor strains are positive), and the CAMP test (El Tor strains are positive) [28].

Environmental. Most dwellings had concrete slab latrines that drained into open sewers. Other households had open privies. Moore swabs (4.0-cm-thick rolls of gauze attached to nylon string) $[29,30]$ were placed in open sewers (near where the household drain entered the sewer) or privies adjacent to 27 dwellings where pairs of children were vaccinated during study 1 . In study 2 , Moore swabs were similarly placed near 70 additional dwellings. The Moore swabs were placed on the evening or night of the same day as vaccination and remained in situ for 36-40 h. After retrieval, the swabs were inoculated into $250 \mathrm{~mL}$ of alkaline peptone enrichment broth and incubated for $6 \mathrm{~h}$ at $37^{\circ} \mathrm{C}$ before subculture onto TCBS agar. Incubation and processing were done in the same manner as for the stool cultures.

\section{Serology}

Venous blood (3-4 $\mathrm{mL}$ ) was collected on the day of vaccination and 9 and 28 days thereafter. Serum Inaba vibriocidal antibody, the primary measurement of immune response, was assayed by microtiter method [31,32], and a fourfold or greater rise in titer was considered significant (i.e., seroconversion) [32]. Serum vibriocidal antibodies are a marker for the elicitation of protective intestinal immune responses against $V$. cholerae $\mathrm{Ol}$ [2]. IgG cholera antitoxin was measured by ELISA in serum specimens diluted 1:50 [33]; if the net optical density (OD) of the prevaccination specimen was $\geqslant 1.00$, sera were tested at $1: 400$ dilution. $A \geqslant 0.20$ rise in net $O D$ of the postvaccination over the prevaccination specimen was considered significant (i.e., seroconversion) [33].

\section{Statistical Tests and Analyses}

Overall rates of adverse reaction and seroconversion were compared by $\chi^{2}$ test with Yates's correction or by Fisher's exact test. Adverse reaction rates and seroconversion rates among the household pairs of children were analyzed by McNemar's matched pairs analysis. Before their analysis, serum Inaba vibriocidal antibody titers were transformed to logarithms to better approximate normality. The relationship between age of subject and occurrence of fever was examined by logistic regression analysis. The relationship between age group $(24-35,36-47$, and 48-59 months) and the fold rise in reciprocal geometric mean titer (GMT) of serum Inaba vibriocidal antibody was examined using analysis of covariance (covariate $=$ day 0 Inaba vibriocidal titer).

\section{Results}

Clinical tolerance. In study 1 , parents from 170 households with age-eligible pairs of children identified during the census expressed interest in participating. On the day of vaccination, 86 pairs of children presented, of which 80 remained eligible after physical examination and age verification and were entered into the study and vaccinated. Of the 160 children ( 80 pairs) who were offered vaccine or placebo suspensions, 75 pairs ingested at least $50 \%$ of the suspension (the cutoff for being considered vaccinated). In fact, 132 (83\%) ingested the full volume. Any child from a pair who ingested the cocktail while the sibling did not was followed to detect adverse reactions. These surveillance data are included in the overall analysis of reactogenicity.

In study 2, parents of 244 age-eligible children expressed interest for their offspring to participate. On the allotted day, 185 children were brought to the vaccination site. Thirty children were judged ineligible by the screening pediatricians, leaving 155 age-eligible children who were offered vaccine; $130(84 \%)$ ingested the entire volume of vaccine or placebo suspension while $149(96 \%)$ ingested at least $50 \%$.

In total, 81 eligible pairs of 24 - to 59 -month-olds were vaccinated (75 during study 1 and 6 during study 2 ) and followed for adverse reactions. Two additional children in study 1 , representing the single members within a pair who ingested the vaccine cocktail (whereas their paired sibling did not), were also kept under clinical surveillance. Finally, in study 2, 139 additional children, 1 per household, received vaccine or placebo.

The overall occurrence of diarrhea, vomiting, abdominal cramps, and fever among these 303 recipients of vaccine or placebo is summarized in table 1 . Neither during the first 3 days of clinical follow-up (when diarrheal reactions were most likely to occur) nor during the entire 9-day period of follow-up were gastrointestinal adverse reactions noted significantly more often in vaccinees. The diarrheal reactions observed in the vaccinees and controls were mild.

Fevers were observed significantly more often in the vac- 
Table 1. Adverse reactions among 24- to 59-month-old Indonesian children who received a single $5 \times 10^{9} \mathrm{cfu}$ dose of CVD $103-\mathrm{HgR}$ or placebo, during 9 days of follow-up after immunization.

\begin{tabular}{|c|c|c|c|c|c|}
\hline \multirow[b]{2}{*}{ Group $(n)$} & \multirow[b]{2}{*}{ Mean age* } & \multicolumn{4}{|c|}{ No. $(\%)$ with reaction } \\
\hline & & Diarrhea & Vomiting & $\begin{array}{l}\text { Abdominal } \\
\text { cramps }\end{array}$ & Fever \\
\hline \multicolumn{6}{|l|}{ All children } \\
\hline Vaccinees (155) & $42.5 \pm 11.1$ & $18(11.6)$ & $16(10.3)$ & $18(11.6)$ & $28(18.1)$ \\
\hline Placebo (148) & $44.3 \pm 11.4$ & $12(8.1)$ & $10(6.7)$ & $13(8.7)$ & $14(9.4)$ \\
\hline$p^{\dagger}$ & 0.17 & 0.69 & 0.37 & 0.53 & 0.045 \\
\hline \multicolumn{6}{|l|}{ Household pairs } \\
\hline Vaccinees $(81)$ & $41.6 \pm 11.6$ & $7(8.6)$ & $5(4.9)$ & $10(12.3)$ & $15(18.5)$ \\
\hline Placebo (8I) & $43.3 \pm 11.9$ & $4(4.9)$ & $4(5.6)$ & $7(8.6)$ & $10(12.3)$ \\
\hline$P^{\ddagger}$ & 0.93 & 0.53 & 1.00 & 0.30 & 0.55 \\
\hline
\end{tabular}

cinees $(18.1 \%)$ than in the placebo recipients $(8.7 \% ; P=$ .045 ; table 1). The fevers were low-grade (the maximum temperature elevation was $39.0^{\circ} \mathrm{C}$ in a vaccinee and $39.4^{\circ} \mathrm{C}$ in a control) and clustered in the youngest children; fevers were recorded in $29 \%$ of children $24-35$ months, $16 \%$ of those 36-47 months, and $10 \%$ of those $48-59$ months of age. The relationship between age and occurrence of fever in the vaccine group was statistically significant (logistic regression, $P$ $=.02$ ). In contrast, the occurrence of fever in placebo recipients was unrelated to age, being observed in $9 \%$ of children aged $24-35$ and $48-59$ months and $12 \%$ of children aged 36-47 months. The fevers demonstrated a curious distribution over time. During the first 5 days of follow-up there was no difference in the occurrence of fever: $14(9 \%)$ of 155 vaccinees versus $12(8.1 \%)$ of 148 controls. However, during the last 4 days of clinical follow-up, fever was observed in 14 vaccinees $(9 \%)$ and only 1 control $(0.7 \% ; P=.002)$. Analysis of the individual cases of fever that occurred among the vaccinees between days 6 and 9 of follow-up after vaccination revealed that 12 of the 14 subjects had clearcut intercurrent infections. These included upper respiratory infections in 8 children, pharyngitis in 2 , and shigellosis in 1.

When the 81 pairs of 24- to 59-month-olds were analyzed separately using a matched pairs analysis, no gastrointestinal or febrile adverse reactions were observed significantly more frequently in vaccinees than in controls (table I).

Immunogenicity. The rate of seroconversion of serum Inaba vibriocidal antibody among vaccinees and placebo recipients for whom blood samples were available for testing is summarized in table 2, as is the GMT before and the peak GMT after vaccination. Overall, $75 \%$ of vaccinees manifested seroconversions; the peak GMT after vaccination was 10-fold above the baseline GMT, and 17\% of children reached reciprocal titers $\geqslant 2560$. Of placebo recipients, $3 \%$ demonstrated seroconversions. The propensity to seroconvert was not related to age (table 2). Although the peak
GMT of vibriocidal antibody rose with age, so did the baseline GMT, undoubtedly reflecting cumulative contact with cholera antigens in this endemic area. The mean fold rise in GMT was highest in the oldest stratum of children within this cohort (analysis of covariance, $P=.018$; table 2). CVD 103-HgR elicited similar seroconversion rates in girls (74\%) and boys ( $76 \%)$.

Baseline serum antitoxin levels were exceedingly high, with an arithmetic mean net $O D$ of 0.92 in the vaccine group and 0.96 in the placebo group. Despite the high baseline titers, significant rises in serum antitoxin were observed in 31 (25\%) of 125 vaccinees and in $7(6 \%)$ of 120 placebo recipients whose sera were available for testing.

Excretion of vaccine strain by vaccinees. Of the 162 vaccinees from whom stool cultures were obtained, the vaccine strain was recovered from only $4(2.5 \%), 2$ in study 1 and 2 in study 2 . One child's stool cultures also yielded $\boldsymbol{V}$. cholerae of a serogroup other than $\mathrm{O} 1$. Wild-type $V$. cholerae $\mathrm{OI}$ strains (biotype El Tor, serotype Ogawa) were isolated from 3 vaccinees ( 1 in study 1 and 2 in study 2 ).

The available classical Inaba $V$. cholerae $\mathrm{Ol}$ isolates were hybridized with DNA probes that detect the cholera toxin A subunit gene and the $\mathrm{Hg}^{++}$gene. The isolates were negative with the former and positive with the latter probe. In contrast, the wild-type El Tor Ogawa isolates hybridized with the toxin probe and were negative with the $\mathrm{Hg}^{++}$probe.

Transmissibility of the vaccine. Transmission of the vaccine strain to placebo recipients and other household contact controls was sought, directly by culturing stool to detect the vaccine strain and indirectly by collecting blood specimens to look for seroconversion of vibriocidal antibody. Neither CVD 103-HgR nor wild-type El Tor V. cholerae was isolated from stool cultures of any of the 81 placebo recipients among the household pairs. CVD $103-\mathrm{HgR}$ was recovered from the stool culture of $1(0.6 \%)$ of 174 other family contacts (a mother). Wild-type $V$. cholerae El Tor Ogawa was isolated 
Table 2. Serum Inaba vibriocidal antibody and serum $\operatorname{IgG}$ antitoxin responses following ingestion of a single $5 \times 10^{9} \mathrm{cfu}$ dose of CVD 103-HgR live oral cholera vaccine or placebo by 24- to 59-month-old Indonesian children.

\begin{tabular}{|c|c|c|c|c|c|c|c|c|}
\hline Group (n) & $\begin{array}{c}\text { Seroconversion } \\
\text { rate }^{*}\end{array}$ & $\begin{array}{c}\text { GMT } \\
\text { before } \\
\text { vaccination }^{\dagger}\end{array}$ & $\begin{array}{l}\text { Peak } \\
\mathrm{GMT}^{\dagger}\end{array}$ & $\begin{array}{c}\text { Rise } \\
\text { in GMT }\end{array}$ & $\begin{array}{c}\text { No. }(\%) \text { with } \\
\text { titer } \\
>2560\end{array}$ & $\begin{array}{c}\text { Seroconversion } \\
\text { rate }^{*}\end{array}$ & $\begin{array}{c}\text { Mean } \\
\text { before } \\
\text { vaccination }{ }^{*}\end{array}$ & $\begin{array}{l}\text { Mean } \\
\text { peak }^{\ddagger}\end{array}$ \\
\hline \multicolumn{9}{|l|}{ Vaccinees } \\
\hline months (4I) & $32(78)$ & $16(2.8 \pm 1.1)$ & $131(4.9 \pm 1.7)$ & 8-fold & $4(10)$ & $8(20)$ & $1.10 \pm 0.67$ & $1.26 \pm 0.60$ \\
\hline $\begin{array}{l}36-47 \\
\text { months }(36)\end{array}$ & $25(69)$ & $24(3.2 \pm 1.2)$ & $176(5.2 \pm 1.7)$ & 7 -fold & $3(8)$ & $9(25)$ & $0.95 \pm 0.44$ & $1.12 \pm 0.49$ \\
\hline $48-59$ & & & & & & & & \\
\hline months (48) & $37(77)$ & $39(3.7 \pm 1.6)$ & $523(6.3 \pm 1.9)$ & 13-fold & $14(29)$ & $14(29)$ & $0.75 \pm 0.52$ & $1.04 \pm 0.67$ \\
\hline $\begin{array}{l}\text { Placebo, } \\
\text { all ages (120) }\end{array}$ & $4(3)$ & $21(3.2 \pm 1.1)$ & $23(3.1 \pm 1.1)$ & - & 0 & $7(6)$ & $0.95 \pm 0.62$ & $0.93 \pm 0.58$ \\
\hline
\end{tabular}

* No. (\%) with 4-fold or greater rises. Postvaccination blood specimens were not available from some vaccinated children.

Reciprocal geometric mean titer ( $\log$ GMT \pm SD).

$\ddagger$ Arithmetic mean net optical density units \pm SD.

from another family contact $(0.6 \%)$. Of the members of the household pairs who received placebo and whose sera were available for testing, only $1(1.5 \%)$ of 68 manifested seroconversions of vibriocidal antibody; similarly, only $4(6 \%)$ of 67 family contacts who provided serum for testing showed significant rises.

Environmental bacteriology to detect CVD $103-\mathrm{HgR}$. Moore swabs from 26 (96\%) of 27 household environments sampled in study 1 yielded non-OI $V$. cholerae, demonstrating the sensitivity of the method. In study $2, V$. cholerae of serogroups other than $\mathrm{Ol}$ were recovered from the environment (wastewater or privy) near 20 (29\%) of 70 dwellings. However, in no instance in either study did a Moore swab culture yield the vaccine strain or $V$. cholerae $\mathrm{Ol}$.

\section{Discussion}

This report summarizes experiences with a single $5 \times 10^{9}$ cfu dose of CVD 103-HgR in 24- to 59-month-old children living in North Jakarta. Because we were evaluating in young children a recombinant live vaccine derived from a known pathogenic parent strain of $V$. cholerae $\mathrm{OI}$, we were particularly concerned about the occurrence of gastrointestinal adverse reactions. It is reassuring that in this randomized, placebo-controlled, double-blind study, neither diarrhea, vomiting, nor abdominal cramps was observed significantly more often among vaccinees. Surprisingly, febrile reactions were noted significantly more often in vaccinees than controls ( $18.1 \%$ vs. $8.1 \% ; P=.045)$. These fevers, which were mostly low-grade, clustered in the youngest children during days 6-9 after vaccination. It is not readily apparent why an attenuated $V$. cholerae $\mathrm{Ol}$ vaccine would be associated with low-grade fevers in this age group, particularly with a late onset. In previous randomized, placebo-controlled trials in adults and 5- to 9-year-old Indonesian children, febrile reactions were not observed [14, 16-23]. Moreover, fever is not a prominent symptom of cholera, although it has been observed in 10\%-20\% of volunteers with experimental cholera [34]. We believe that this is a chance finding not biologically attributable to vaccine, since careful examination of the clinical records of each of the children who manifested fever during the latter third of the clinical follow-up (when fever was seen more often in vaccinees) revealed an intercurrent infection in 12 of 14 vaccinees. The fevers in vaccinees were not correlated with diarrhea $(P=.46)$ or other gastrointestinal adverse effects. A similarly designed reactogenicity and immunogenicity study of CVD $103-\mathrm{HgR}$ in 24- to 59month-olds that was recently completed in Costa Rica showed no difference in the rate of occurrence of fever between vaccinees and controls (unpublished data).

A single dose of CVD 103-HgR was quite immunogenic. Seventy-five percent of vaccinees manifested significant rises in vibriocidal antibody (the best correlate of protection) [2, 35-38], and overall the vaccinees demonstrated a mean 10fold rise in GMT over baseline (table 2). For the purpose of comparison, it is of interest to note that among children 2-5 years of age in Bangladesh who ingested three doses of inactivated whole cell $V$. cholerae $\mathrm{Ol}$ vaccine (either alone or combined with B subunit), $36 \%$ manifested 4 -fold rises in serum Inaba vibriocidal antibody and overall they exhibited a mean 4-fold increase in titer over baseline; after a single dose of vaccine, only $18 \%$ exhibited 4-fold rises [26].

A characteristic feature of CVD $103-\mathrm{HgR}$ live oral cholera vaccine is that while it elicits excellent immune responses, it is minimally excreted. In these studies of those 24-59 months old, the vaccine strain was recovered from stool cul- 
tures of only $2.5 \%$ of vaccinees. With such minimal excretion one would not anticipate frequent secondary transmission of the vaccine strain. This study was designed to incorporate conditions that would favor transmissibility: for example, the study was carried out in 24- to 59-month-olds, an age group in which fecal transmission occurs readily because of primitive personal hygiene practices, even in industrialized settings with high levels of sanitation [39, 40]; moreover, dwellings in the study villages typically do not have piped water or sewage disposal. Despite these conditions, neither by stool culture nor by seroconversion of vibriocidal antibody was there much evidence of secondary transmission of the vaccine strain to contacts. Since El Tor cholera is endemic in North Jakarta, one cannot exclude the possibility that some of the few seroconversions of vibriocidal antibody observed in placebo recipients and family contacts were due to ingestion of wild-type $V$. cholerae $\mathrm{Ol}$. Even if all the seroconversions among contacts were due to the live vaccine, this would represent a secondary transmission rate of only 6\% under conditions highly favorable for such transfer.

Obtaining information on transmissibility of the vaccine strain was critical in order to design a large-scale field trial to determine the efficacy of a single dose of CVD 103-HgR in preventing cholera under conditions of natural challenge in North Jakarta. Earlier field trials elsewhere that examined the efficacy of parenteral and oral inactivated vaccines were designed so that individual subjects were randomly allocated to receive vaccine or placebo [41-45]. If CVD I03-HgR live cholera vaccine were readily transmitted to contacts, as is the Sabin attenuated poliomyelitis vaccine [46], it would be incorrect to design a field trial based on random allocation of individuals. With a readily transmissible vaccine strain, many placebo recipients inadvertently would be immunized; as a result, the incidence of cholera in the placebo group would be falsely low and this, in turn, would lower the calculated vaccine efficacy. Rather, to field test the efficacy of a readily transmitted live oral cholera strain, an innovative design would be required in which the unit of random allocation would be villages or extended households, not individuals. On the basis of the apparent low level of transmission of CVD 103-HgR even among preschool children, a largescale, randomized, placebo-controlled, double-masked field trial, which follows the classic study design by which earlier cholera vaccines were tested, was initiated in June 1993 in 66,000 pediatric and adult subjects in North Jakarta. This World Health Organization-sponsored field trial will measure the efficacy of a single dose of CVD $103-\mathrm{HgR}$ in preventing cholera in this high-risk area.

Recombinant DNA technology has revolutionized vaccine development, resulting in new or improved vaccines against many infectious diseases [47]. Nevertheless, a segment of the public and the scientific community remains suspicious of the products of recombinant DNA technology and of the release into the environment of genetically engi- neered strains, including vaccines [48-52]. Although the US Food and Drug Administration does not regulate genetically engineered vaccines any differently than it does live vaccines prepared by nonrecombinant means such as chemical mutagenesis, there are advantages to using a live recombinant vaccine such as CVD 103- $\mathrm{HgR}$ that is not heavily excreted and that does not readily enter or persist in the environment. The sensitivity and utility of Moore swab environmental sampling to isolate Salmonella typhi $[30,53,54]$ and $V$. cholerae 01 [29] from fecally contaminated surface waters is well-established. Moore swabs did not recover CVD 103$\mathrm{HgR}$ from the environment around any of the 97 North Jakarta households sampled.

The international, multiagency Children's Vaccine Initiative [55] is promulgating the concept of immunizing infants by the oral route with single-dose, multiantigen vaccines. The remarkable immunogenicity of a single dose of CVD $103-\mathrm{HgR}$ in pediatric populations in less-developed countries [22], and its efficacy thus far in experimental challenge studies in volunteers [14, 19], adds optimism for the generic notion of future multivalent single-dose oral vaccines. In this context, CVD 103-HgR itself is being investigated as a potential live vector vaccine to express foreign antigens [56].

\section{Acknowledgments}

We thank the many individuals from Jakarta Municipality, Maramis Hisham and staff of the Infectious Diseases Hospital, the Departments of Pediatrics, Microbiology, and Immunology of the University of Indonesia, the Indonesian National Institute of Health, and US Naval Medical Research Unit No. 2 who contributed to this project and Kimi F. Y. Lin and Kenneth Bart of the National Vaccine Program. We thank Dale Spriggs and Jorge Flores, National Institute of Allergy and Infectious Diseases, National Institutes of Health, Juan Urrutia, Pan American Health Organization, and Roger Glass, Centers for Disease Control, for serving as the Data Safety Monitoring Committee.

\section{References}

1. Swerdlow DI, Mintz ED, Rodriguez M, et al. Waterborne transmission of epidemic cholera in Trujillo, Peru: lessons for a continent at risk. Lancet 1992;340:28-33.

2. Levine MM, Pierce NF. Immunity and vaccine development. In: Greenough WB III, Barua D, eds. Cholera. New York: Plenum Press, 1992:285-327.

3. Feeley JC, Gangarosa EJ. Field trials of cholera vaccine. In: Cholera and related diarrhoeas. 43rd Nobel Symposium, Stockholm, 1978. Basel: Karger, 1980:204-10.

4. Glass RI, Claeson M, Blake PA, Waldman RJ, Pierce NF. Cholera in Africa: lessons on transmission and control for Latin America. Lancet 1991;338:791-5.

5. World Health Organization. Guidelines for cholera control. Geneva: WHO, 1991;WHO/CDD/SER/80.4, rev 2.

6. Diarrhoeal Diseases Control Programme, World Health Organization. Biomedical and epidemiological research priorities of global scien- 
tific working groups. Geneva: WHO, 1987;WHO/CDD/SER/86.8, rev 1.

7. Levine MM. Vaccines against enteric infections. Lancet 1990;335: 958-61.

8. Levine MM. Kaper JB. Live vaccines against cholera: an up-date. Vaccine 1993:11:207-12.

9. Clemens JD, Harris JR, Sack DA, et al. Field trial of oral cholera vaccines in Bangladesh: results of one year of follow-up. J Infect Dis 1988:158:60-9.

10. Clemens JD, Sack DA. Harris JR, et al. Field trial of oral cholera vaccines in Bangladesh: results from three year follow-up. Lancet 1990;335:270-3.

11. Kaper JB, Lockman H. Baldini MM, Levine MM. Recombinant live oral cholera vaccine. Biotechnology 1984;2:345-9.

12. Mekalanos JJ, Swartz SJ. Pearson GDN, et al. Cholera toxin genes: nucleotide sequence, deletion analysis, and vaccine development. Nature 1983:306:551-7.

13. Kaper JB, Levine MM. Recombinant attenuated Vibrio cholerae strains used as live oral vaccines. Res Microbiol 1990;141:901-6.

14. Levine MM, Kaper JB, Herrington D, et al. Safety, immunogenicity, and efficacy of recombinant live oral cholera vaccine CVD 103 and CVD 103-HgR. Lancet 1988;2:467-70.

15. Ketley JM, Michalski J. Galen J, Levine MM, Kaper JB. Construction of genetically-marked Vibrio cholerae $\mathrm{O} I$ vaccine strains. FEMS Microbiol Lett 1993 (in press)

16. Cryz SC Jr, Levine MM, Kaper JB. Furer E. Althaus B. Randomized double-blind placebo-controlled trial to evaluate the safety and immunogenicity of the live oral cholera vaccine strain CVD 103-HgR in Swiss adults. Vaccine 1990;8:577-80.

17. Kotloff K. Wasserman SS, O'Donnell S, Losonsky GA, Cryz SJ, Levine MM. Safety and immunogenicity in North Americans of a single dose of live oral cholera vaccine CVD $103-\mathrm{HgR}$ : results of a randomized, placebo-controlled, double-blind cross-over trial. Infect Immun 1992:60:4430-2.

18. Cryz S, Levine MM, Losonsky G, Kaper JB. Althaus B. Safety and immunogenicity of a booster dose of Vibvio cholerae CVD $103-\mathrm{HgR}$ live oral cholera vaccine in Swiss adults. Infect Immun 1992; 60:3916-7

19. Tacket CO. Losonsky G. Nataro J. et al. Rapid onset and duration of protective immunity in challenged volunteers after vaccination with live oral cholera vaccine CVD 103-HgR. J Infect Dis 1992;166:83741.

20. Migasena S, Pitisuttitham P. Prayurahong B, et al. Preliminary assessment of the safety and immunogenicity of live oral cholera vaccine strain CVD 103- $\mathrm{HgR}$ in healthy Thai adults. Infect Immun 1989:57:3261-4.

21. Su-Arehawatana P. Singharaj P. Taylor D. et al. Safety and immunogenicity of different immunization regimens of CVD $103-\mathrm{HgR}$ live oral cholera vaccine in soldiers and civilians in Thailand. $J$ Infect Dis 1992;165:1042-8.

22. Suharyono. Simanjuntak C, Witham $N$, et al. Safety and immunogenicity of single-dose live oral cholera vaccine CVD 103- $\mathrm{HgR}$ in 5-9year-old Indonesian children. Lancet 1992;340:689-94.

23. Gotuzzo E. Penny M. Seas C, et al. The safety, immunogenicity, and excretion pattern of single-dose live oral cholera vaccine, CVD 103hgR in Peruvian adults of high and low socioeconomic level. Infect Immun 1993 (in press).

24. Simanjuntak CH. Hasibuan MA. Siegar LO. Koiman I. Etiologi mikrobiologis penyakit diare akut. Health Studies Indonesia 1983;1 1:1-9.

25. Simanjuntak $\mathbf{C H}$, Harjining $\mathbf{S}$, Hasibuan MA, Pujarwoto, Koiman I. Laboratory aspects of gastrointestinal infections in Indonesia: 19801985. In: Proceedings of the 14th SEAMIC Workshop, Gastrointestinal Infections in Southeast Asia. Tokyo: SEAMIC, 1988:23-31.

26. Clemens JD, Stanton BF. Chakraborty J, et al. B subunit-whole cell and whole cell-only oral vaccines against cholera: studies on reactogenicity and immunogenicity. $J$ Infect Dis 1987;155:79-85.

27. Farmer JJ III, Hickman-Brenner FW, Kelly MT. Vibrio. In: Lennette EH. Balows A. Hausler WJ Jr, Shadomy J, eds. Manual of clinical microbiology. 4th ed. Washington, DC: American Society for Microbiology, 1985:282-301

28. Lesmana M, Rockhill RC. A CAMP phenomenon between $V$. cholerae biotype El Tor and staphylococcal $\beta$-hemolysin. Southeast Asian J Trop Med Public Health 1985; 16:261-4.

29. Barrett TJ, Blake PA, Morris GK, Puhr ND, Bradford HR, Wells JG. Use of Moore swabs for isolating Vibrio cholerae from sewage. J Clin Microbiol 1980;11:385-8.

30. Sears SD, Ferreccio C, Levine MM. Sensitivity of Moore sewer swabs for isolating Salmonella typhi. Appl Environ Microbiol 1986;51: 425-6.

31. Benenson AS, Saad A, Mosley WH. Serological studies in cholera. 2. The vibriocidal antibody response of cholera patients determined by a microtechnique. Bull WHO 1968;38:277-85.

32. Clements ML, Levine MM, Young CR, et al. Magnitude, kinetics and duration of vibriocidal responses in North Americans following ingestion of Vibrio cholerae. J Infect Dis 1982;145:465-73.

33. Levine MM, Young CR, Black RE, Takeda Y, Finkelstein RA. Enzyme-linked immunosorbent assay to measure antibodies to purified heat-labile enterotoxins from human and porcine strains of Escherichia coli and to cholera toxin: application in serodiagnosis and seroepidemiology. J Clin Microbiol 1985;21:174-9.

34. Levine MM, Black RE, Clements ML, Nalin DR, Cisneros L, Finke\}stein RA. Volunteer studies in development of vaccines against cholera and enterotoxigenic Escherichia coli: a review. In: Holme T, Holmgren J, eds. Acute enteric infections in children: new prospects for treatment and prevention. Amsterdam: Elsevier, 1981:443-59.

35. Mosley WH. Beneson AS. Barui R. A serological survey for cholera antibodies in rural cast Pakistan. I. The distribution of antibody in the control population of a cholera vaccine field-trial area and the relation of antibody titer to the pattern of endemic cholera. Bull WHO 1968:38:327-34.

36. Mosley WH. Ahmad S. Benenson AS. Ahmed A. The relationship of vibriocidal antibody titre to susceptibility to cholera in family contacts of cholera patients. Bull WHO 1968;38:777-85.

37. Glass RI. Svennerholm AM, Khan MR, Huda S, Huq MI, Holmgren J. Seroepidemiological studies of EI Tor cholera in Bangladesh: association of serum antibody levels with protection. J Infect Dis 1985; 151:236-42.

38. Clements JD, Van Loon F, Sack DA, et al. Field trial of oral cholera vaccines in Bangladesh: serum vibriocidal and antitoxic antibodies as markers of the risk of cholera. J Infect Dis 1991;163:1235-42.

39. Weissman JB, Gangarosa EJ, Schmerler A, Marier RL, Lewis JN. Shigellosis in day-care centers. Lancet 1975:1:88-90.

40. Pickering LK, Bartlett AV, Woodward WE. Acute infectious diarrhea among children in daycare: epidemiology and control. Rev Infect Dis 1986;8:539-47.

41. Clemens JD, Sack DA, Harris JR, et al. Field trial of oral cholera vaccines in Bangladesh. Lancet 1986;2:124-7.

42. Mosley WH, McCormack WM. Ahmed A, et al. Report of the 1966-67 cholera vaccine field trial in rural East Pakistan. 1. Study design and results of the first year of observation. Bull WHO 1969;40:177-85.

43. Philippines Cholera Committee. A controlled field trial of the effectiveness of cholera and cholera El Tor vaccines in the Philippines. Bull WHO 1965;32:603-25.

44. Saroso JS, Bahrawi W, Witjaksono $\mathrm{H}$, et al. A controlled field trial of plain and aluminum hydroxide-adsorbed cholera vaccines in Surabaya, Indonesia, during 1973-75. Bull WHO 1978;56:619-27.

45. Das Gupta A, Sinha R, Shrivastava DL, et al. Controlled field trial of 
the effectiveness of cholera and cholera El Tor vaccines in Calcutta. Bull WHO 1967;37:371-85.

46. Sabin AB. Oral poliomyelitis vaccine. History of its development and prospects for eradication of poliomyelitis. JAMA 1965;194:130-4.

47. Woodrow G. An overview of biotechnology as applied to vaccine development. In: Woodrow G, Levine MM, eds. New generation vaccines. New York: Marcel Dekker, 1990:31-42.

48. Fox JL. Anticipating deliberate release. A growing intellectual industry. ASM News 1985;51:619-20.

49. Fox JL. Contemplating suicide genes in the environment. Conditional lethal mutations may minimize risk when engineered microbes are released into the environment. ASM News 1989;55:259-61.

50. Fox JL. Rabies vaccine field test-pending. An engineered vaccine for use on wild animals seems ready for testing, but state health officials and environmentalists are seeking delays. ASM News 1989;55: $355-7$.
51. Current Topics. NIH RAC eases deliberate release restrictions. ASM News 1987:53:195-6.

52. Tieder JM, Colwell RK, Grossman YL, et al. The planned introduction of genetically-engineered organisms: ecological considerations and recommendations. Ecology 1989;70:298-316.

53. Moore B. Perry EL, Chard ST. A survey by the sewage swab method of latent enteric infections in an urban area. J Hyg 1952;50:137-56.

54. Sears SD, Ferreccio C, Levine MM, et al. The use of Moore swabs for isolation of Salmonella typhi from irrigation water in Santiago. Chile. $J$ Infect Dis 1984;149:640-2.

55. Robbins A, Freeman P. The Children's Vaccine Initiative. Lancet 1991;338:1006-7.

56. Viret J, Cryz S, Lang A, Favre D. Molecular cloning and characterization of the genetic determinants that express the complete Shigella serotype (Shigella sonnei) lipopolysaccharide in heterologous live attenuated vaccine strains. Mol Microbiol 1993;7:239-52. 